\title{
Mammographically Detected Breast Arterial Calcification-A Marker of Coronary Artery Disease in Women
}

\author{
Padmasri Gorantla ${ }^{1}$ Anu Kapoor ${ }^{1} \quad$ Jyotsna Maddury ${ }^{2}$ \\ ${ }^{1}$ Department of Radiology and Imageology, Nizam's Institute of \\ Medical Sciences, Punjagutta, Hyderabad, Telangana, India \\ 2Department of Cardiology, Nizam's Institute of Medical Sciences, \\ Punjagutta, Hyderabad, Telangana, India \\ Address for correspondence Padmasri Gorantla, MD, Department \\ of Radiology and Imageology, Nizam's Institute of Medical Sciences, \\ Punjagutta, Hyderabad, TS 500082, India \\ (e-mail: dr.padmasri28@gmail.com).
}

Indian J Cardiovasc Dis Women-WINCARS 2017;2:82-85

\begin{abstract}
Background and Aim Coronary artery disease (CAD) resulting from atherosclerosis is one of the major causes of mortality and morbidity worldwide and is still a leading cause of death in women older than 40 years. The gold standard for diagnosis of CAD is by coronary angiography (CAG). However, this procedure is both invasive and expensive and thus is not suitable as a screening tool in asymptomatic individuals. Like coronary calcification, atherosclerotic vascular calcification involving small-tomedium-sized arteries is also observed in the breast. Modern mammographic equipment is very sensitive in the detection of microcalcifications. The aim of this study is to study the relationship between mammographically detected breast arterial calcification (BAC) and CAD and to evaluate the role of BAC as a marker for CAD.

Methods Twenty female patients older than 40 years who had undergone CAG for suspected CAD were included in the study. Screening mammograms were performed and analyzed for the presence of BAC. The results were analyzed for correlation between severity of BAC and CAD.

Keywords

- mammography

- breast

- calcification

- coronary artery disease

-women

Results Twenty patients with mean age of 56.45 (age range: 40-68) were evaluated. BACs were found in $60 \%$ of these cases with a peak age group of 56 to 60 years. BAC was bilateral (92\%) in most cases. CAG reports were positive for CAD in $45 \%$ of patients. The sensitivity of $B A C$ in predicting CAD was $77.7 \%$ with a specificity of $54.5 \%$, positive predictive value (PPV) of $58.3 \%$, and negative predictive value (NPV) of $75 \%$.

Conclusion Screening mammography has a potential to serve as a noninvasive tool for early detection of CAD in asymptomatic women. Larger population-based studies with controls will be required to establish the utility of this screening tool.
\end{abstract}

\section{Introduction}

Coronary artery disease (CAD) resulting from atherosclerosis is one of the major causes of mortality and morbidity worldwide. There are distinct sex-based differences in etiology, pathophysiology, and manifestations of symptoms of CAD, but it is still a leading cause of death in women older than 40 years. ${ }^{1-4}$

For many asymptomatic individuals with CAD, the first manifestation of underlying disease is often an unexpected

Dol https://doi.org/ 10.1055/s-0038-1622963. acute myocardial infarction or sudden death. Most cardiac events are experienced by individuals thought to be at low to intermediate risk. This has resulted in considerable interest in new methods that would allow for early detection of individuals at increased risk for these events.

The gold standard for diagnosis of CAD is coronary angiography (CAG). However, this procedure is both invasive and expensive, and thus is not suitable as a screening tool in asymptomatic individuals. Other noninvasive methods such as the treadmill test (TMT) and stress echocardiography

Copyright $@ 2017$ Women in Cardiology and Related Sciences
License terms

(®) $\Theta \circledast$ 
depend on provoking ischemia and are not sensitive enough in the early stages of coronary atherosclerosis. In addition, it has been observed that women show poor exercise performance when undergoing TMT, ${ }^{5}$ resulting in a high number of incomplete or nonconclusive results as reported in literature. ${ }^{6}$

Like coronary calcification, atherosclerotic vascular calcification involving small-to-medium-sized arteries is also observed in the breast. Modern mammographic machines are very sensitive in the detection of microcalcifications and can detect calcifications as small as $100 \mu \mathrm{m}$ in size. Breast arterial calcification (BAC), as seen on mammograms, represents medial arterial calcification or Monckeberg's calcification and is different from intimal calcification. ${ }^{7}$

BAC has been proposed as potential markers for CAD, but its reliability and predictive value remain controversial. ${ }^{8-11}$ Several studies have reported the presence of BAC at mammography as a marker for increased risk of future cardiovascular disease-related death in women older than 50 years. However, there is no substantial convincing evidence that links BAC to CAD lesions seen at angiography.

Furthermore, there are no studies reported from the Indian subcontinent so far that correlate BAC and cardiovascular risk. If a significant correlation is found between BAC and CAD, it would be of major public health benefit and mammography then can be proposed as a single screening tool for diagnosis of breast cancer as well as CAD.

The main aim of this study is to study the relationship between mammographically detected BAC and CAD and to evaluate the role of BAC as a marker for CAD in Indian women.

\section{Materials and Methods}

Following approval from the institutional ethics committee, this prospective, cross-sectional study was undertaken in the authors' institute over a period of 3 months from May to July 2017.

Twenty women older than 40 years who underwent CAG for suspected CAD were evaluated using screening mammography, if they have not already had a mammogram within 1 year prior to CAG. Written and informed consent was taken from all the patients prior to their inclusion in the study. Women with prior history of breast trauma/breast

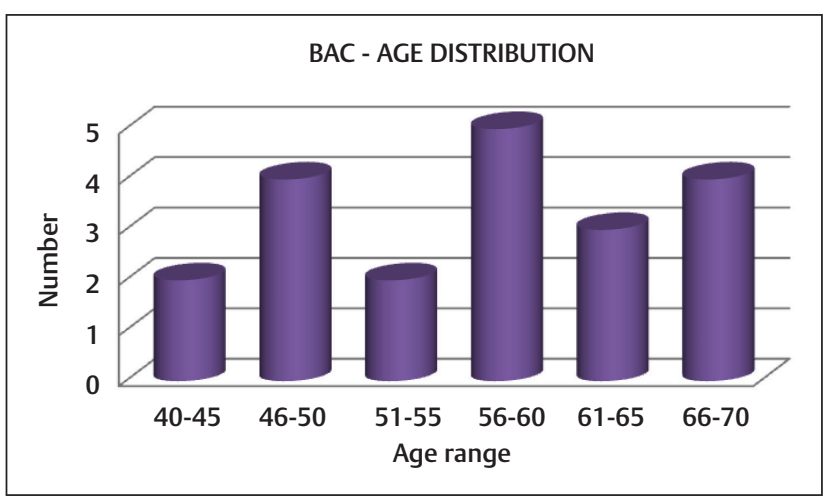

Fig. 1 Representative chart of age distribution of breast arterial calcification (BAC). neoplasm or any other calcium-related metabolic disorders were excluded from the study.

Screening mammography was performed on analog mammography system (Lilyum, Metaltronica Spa), and images were obtained using Capsula X, (Fuji CR system, Fujifilm Corp.).

Mediolateral oblique (MLO) and craniocaudal (CC) views of both breasts were obtained for all cases. The mammographs were reviewed and reported by an experienced radiologist, who was blinded to the clinical data and findings of CAG. The number of calcified vessels as well as laterality of BAC was noted, and BAC was graded as per the described criteria.

These observations and results were analyzed for assessing the relative frequency of CAD in women with and without BAC and for studying the correlation between severity of $\mathrm{BAC}$ and CAD.

\section{Results}

Total 20 cases were included in the study with a mean age of the patients being 56.45 (age range: 40-68) (-Fig. 1). Of the 20 patients, BACs were detected in $12(60 \%)$ patients and CAG reports were positive for CAD in $8(45 \%)$ (-Table $\mathbf{1})$.

BAC was bilateral in most cases (92\%) (-Fig. 2). In this study, the sensitivity of BAC in predicting CAD was $77.7 \%$ with a specificity of $54.5 \%$, positive predictive value (PPV) of $58.3 \%$, and negative predictive value (NPV) of 75\% (- Table 2 ).

\section{Discussion}

Like coronary calcification, atherosclerotic vascular calcification involving small-to-medium-sized arteries is also observed in the breast. Modern mammographic machines are very sensitive in the detection of microcalcifications and can detect calcifications as small as $100 \mu \mathrm{m}$ in size. BAC has been proposed as potential markers for CAD, but its reliability and predictive value remain controversial. Several studies have reported the presence of BAC at mammography as a marker for increased risk of future cardiovascular diseaserelated death in women older than 50 years. In this study, of the 20 patients, BACs were detected in $12(60 \%)$ patients and CAG reports were positive for CAD in 8 (45\%).

Mammographs were considered positive for BAC if they showed the following patterns of calcifications in any one plane of imaging:

- A classic railroad track pattern of linear and parallel calcified lines with amorphous calcification between them.

Table 1 Incidence of breast arterial calcification in CAD

\begin{tabular}{|l|l|l|l|}
\hline & $\begin{array}{l}\text { CAG } \\
\text { positive }\end{array}$ & $\begin{array}{l}\text { CAG } \\
\text { negative }\end{array}$ & Total \\
\hline BAC positive & 7 & 5 & 12 \\
\hline BAC negative & 2 & 6 & 8 \\
\hline Total & 9 & 11 & 20 \\
\hline
\end{tabular}

BAC, breast arterial calcification; CAD, coronary artery disease; CAG, coronary angiography. 


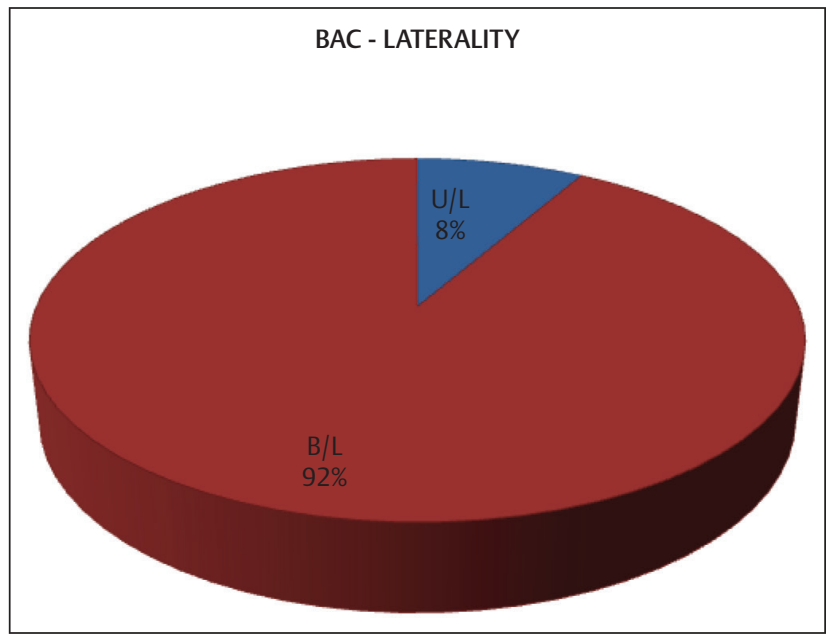

Fig. 2 Pie diagram showing laterality of breast arterial calcification (BAC). B/L, bilateral; U/L, unilateral.

- Or any visible linear calcium deposits along the periphery of tapered structures that are distinct from breast ducts and have a configuration typical of arteries and are at least $1 \mathrm{~mm}$ in length.

The severity of BAC was graded as follows:

A. Absent-no vascular calcifications.

B. Slight-arteries with little calcification with distances greater than $10 \mathrm{~mm}$ between calcified areas.

C. Moderate-arteries clearly outlined by calcifications over a considerable proportion of their course.

D. Severe-arteries extensively affected; seen to have almost continuous columns of calcification, with at least two branches also visible (-Fig. 3 ).
Table 2 Sensitivity, specificity, PPV, and NPV of BAC in detecting CAD

\begin{tabular}{|l|l|}
\hline & Value (\%) \\
\hline Sensitivity & 77.78 \\
\hline Specificity & 54.55 \\
\hline PPV & 58.33 \\
\hline NPV & 75 \\
\hline
\end{tabular}

Abbreviations: BAC, breast arterial calcification; CAD, coronary artery disease; NPV, negative predictive value; PPV, positive predictive value.

In this analysis, most of the BACs were bilateral (92\%), and the peak age group in which BACs were detected was 56 to 60 years. In this study, the sensitivity of BAC in predicting CAD was $77.7 \%$ with a specificity of $54.5 \%$, PPV of $58.3 \%$, and NPV of 75\% (- Table 2).

No statistically significant correlation between the presence of BAC and CAD could be derived due to the small sample size. There are few limitations to this study. First, large sample size is required to obtain a statistically significant data. Second, other associated comorbidities and risk factors associated with BAC were not considered in the study.

To conclude, screening mammography has a potential to serve as a noninvasive tool for early detection of CAD in asymptomatic women. Larger population-based studies with controls are required to establish the utility of this screening tool.

\section{Conflict of Interest}

None.

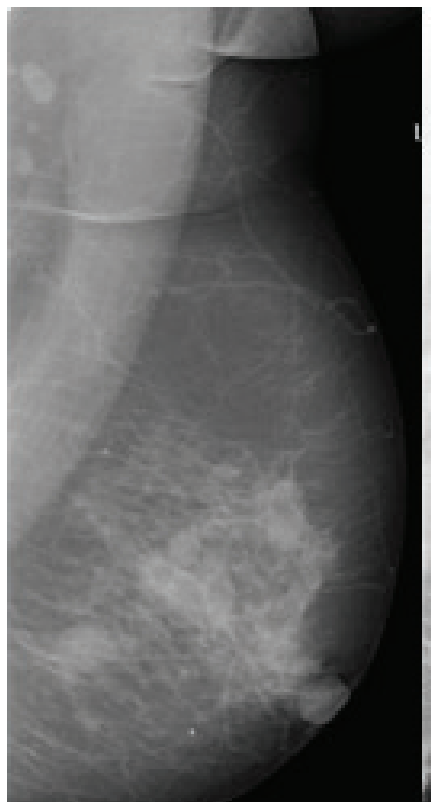

A

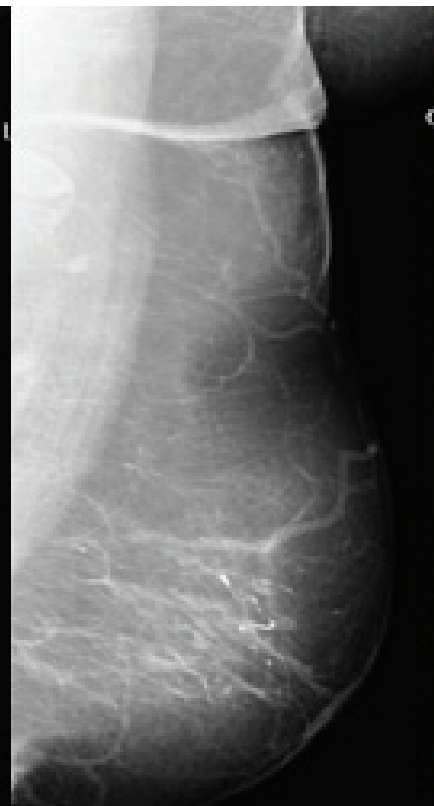

B

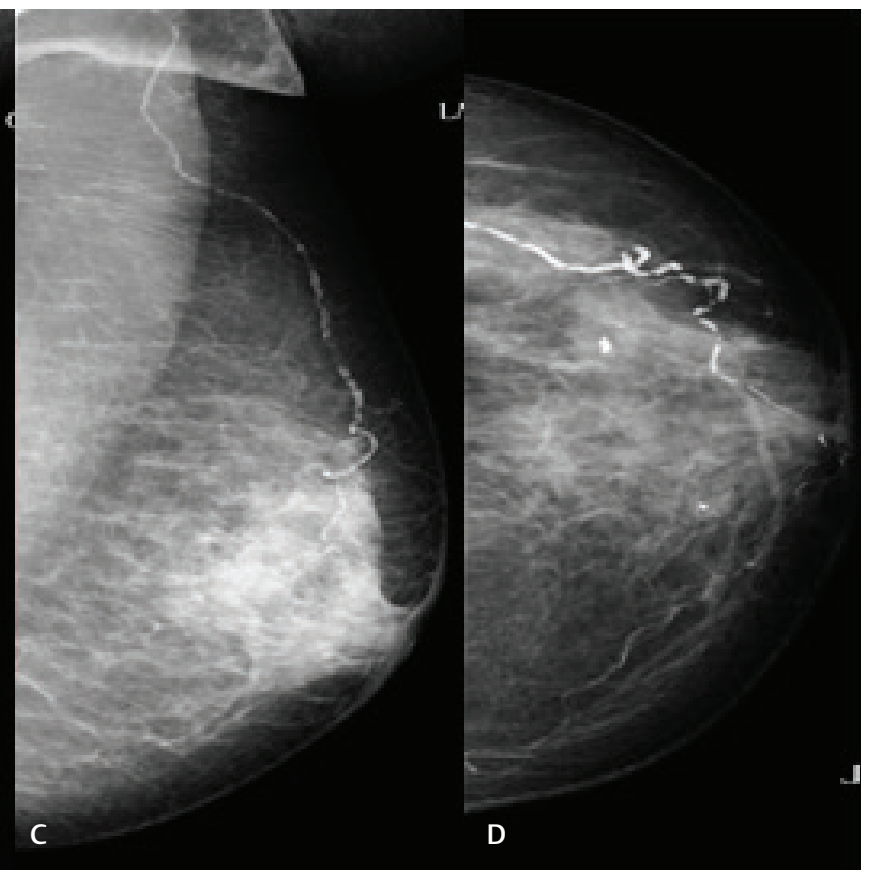

Fig. 3 Grading of severity of breast arterial calcifications. (A) Absent. (B) Slight. (C) Moderate. (D) Severe calcifications. 


\section{Informed Consent}

Informed consent was obtained from all individual participants included in the study.

\section{Ethical Approval}

All procedures performed in studies involving human participants were in accordance with the ethical standards of the institutional and/or national research committee and with the 1964 Helsinki declaration and its later amendments or comparable ethical standards.

This article does not contain any studies with animals performed by any of the authors.

\section{References}

1 Rosamond W, Flegal K, Friday G, et al; American Heart Association Statistics Committee and Stroke Statistics Subcommittee. Heart disease and stroke statistics-2007 update: a report from the American Heart Association Statistics Committee and Stroke Statistics Subcommittee. Circulation 2007;115(5):e69-e171

2 Quyyumi AA. Women and ischemic heart disease: pathophysiologic implications from the Women's Ischemia Syndrome Evaluation (WISE) study and future research steps. J Am Coll Cardiol 2006;47(3, Suppl):S66-S71

3 Pepine CJ. Ischemic heart disease in women. J Am Coll Cardiol 2006;47(3, Suppl):S1-S3

4 Bairey Merz CN, Shaw LJ, Reis SE, et al; WISE Investigators. Insights from the NHLBI-sponsored Women's Ischemia
Syndrome Evaluation (WISE) study: part II: gender differences in presentation, diagnosis, and outcome with regard to gender-based pathophysiology of atherosclerosis and macrovascular and microvascular coronary disease. J Am Coll Cardiol 2006;47(3, suppl):S21-S29

5 Roger VL, Jacobsen SJ, Pellikka PA, Miller TD, Bailey KR, Gersh $B J$. Gender differences in use of stress testing and coronary heart disease mortality: a population-based study in Olmsted County, Minnesota. J Am Coll Cardiol 1998;32(2):345-352

6 Shah SF, Meo SA. Usefulness of standard treadmill stress testing in women. J Pak Med Assoc 2009;59(4):197-200

7 Kim H, Greenberg JS, Javitt MC. Breast calcifications due to Mönckeberg medial calcific sclerosis. Radiographics 1999;19(5):1401-1403

8 Moradi M, Adibi A, Abedi M. Relationship between breast arterial calcification on mammography with CT Calcium scoring and coronary CT angiography results. Adv Biomed Res 2014;3:79

9 Kataoka M, Warren R, Luben R, et al. How predictive is breast arterial calcification of cardiovascular disease and risk factors when found at screening mammography? AJR Am J Roentgenol 2006;187(1):73-80

10 Iribarren C, Go AS, Tolstykh I, Sidney S, Spring DB. Breast vascular calcification and risk of coronary heart disease, stroke, and heart failure. J Womens Health (Larchmt) 2004; 13(4):381-389, discussion 390-392

11 Oliveira EL, Freitas-Junior R, Afiune-Neto A, Murta EF, Ferro JE, Melo AF. Vascular calcifications seen on mammography: an independent factor indicating coronary artery disease. Clinics (Sao Paulo) 2009;64(8):763-767 